\title{
Preparation and Characterization of Ginger Lipid-derived Nanoparticles for Colon-targeted siRNA Delivery Junsik Sung ${ }^{1, *}$, Chunhua Yang ${ }^{1}$, James F. Collins ${ }^{3}$ and Didier Merlin ${ }^{1,2}$
}

${ }^{1}$ Institute for Biomedical Science, Center for Diagnostics and Therapeutics, Georgia State University, Atlanta, GA, USA; ${ }^{2}$ Atlanta Veterans Affairs Medical Center, Decatur, GA, USA; ${ }^{3}$ Food Science \& Human Nutrition Department, University of Florida, Gainesville, FL, USA

*For correspondence: jsung9@gsu.edu

[Abstract] Synthetic nanoparticle-based drug delivery system is widely known for its ability to increase the efficacy and specificity of loaded drugs, but it often suffers from relatively higher immunotoxicity and higher costs as compared to traditional drug formulations. Contrarily, plant-derived nanoparticles appear to be free from these limitations of synthetic nanoparticles; they are naturally occurring biocompatible vesicles that do not generate immunotoxicity and are easy to obtain. Additionally, lipids isolated from plant-derived nanoparticles have shown the capability of assembling themselves to spherical nano-sized liposomal particles. Herein, we employ lipids extracted from ginger-derived nanoparticles and load them with therapeutic siRNA (CD98-siRNA) to create CD98-siRNA/ginger-lipid nanoparticles. Characterization of the CD98-siRNA/ginger-lipid nanoparticles showed that they present a spherical shape, with a diameter of around $189.5 \mathrm{~nm}$. The surface zeta potential of the nanoparticles varies from -18.1 to $-18.4 \mathrm{mV}$. Furthermore, in recent research, the CD98-siRNA/ginger-lipid nanoparticles have shown specific colon targeting capability and excellent anti-inflammatory efficacy in a Dextran Sodium Sulfate (DSS) induced mouse model of colitis.

Keywords: Ginger-derived nanoparticles, Thin-film hydration, siRNA delivery, Anti-inflammatory, Colontargeted

[Background] Small interference RNAs (siRNAs) are a type of promising therapeutic agents that can treat various diseases by silencing the abnormally upregulated messenger RNAs (mRNAs) (Nikam and Gore, 2018). Despite the effectiveness of siRNAs, the safe and efficient delivery of the siRNAs to the therapeutic targets is still a challenging task (Tatiparti et al., 2017). Studies have shown that artificially synthesized nanoparticles can be used to target low doses of siRNAs to specific colonic cell types (e.g., epithelial cells and macrophages) (Zhang et al., 2016b). However, artificially synthetic nanoparticles have several limitations, such as potential in vivo immune toxicity and formidable production cost. What is currently needed is a safer and more economical platform for siRNA-based drug delivery.

Our laboratory and others have recently shown that plant-derived nanoparticles are capable of specifically target the colon, and these naturally occurring nanoparticles are safer and cheaper than synthetic nanoparticles (Zhang et al., 2016a). Additionally, we can extract lipids from these plant-derived nanoparticles and reassemble them to siRNA-incorporated nanoparticles, creating a colon-targeting siRNA/lipid nanoparticle (Zhang et al., 2017). In the following protocol, we will use ginger-derived 
nanoparticles and CD98-siRNA as starting materials to demonstrate the process of making CD98 siRNA nanoparticles (CD98-siRNA/ginger-lipid). The nanoparticles prepared in this protocol have shown its effectiveness in treating ulcerative colitis in recent research (Zhang et al., 2017).

\section{Materials and Reagents}

1. Pipette tips:

0.1-10 $\mu \mathrm{l}$ (Sorenson Bioscience, catalog number: 70600)

1-200 $\mu \mathrm{l}$ (Sorenson Bioscience, catalog number: 70520)

100-1,000 $\mu \mathrm{l}$ (Sorenson Bioscience, catalog number: 70540)

2. $1.5 \mathrm{ml}$ Eppendorf Safe-Lock tubes (Eppendorf, catalog number: 022363204)

3. $22 \mathrm{ml}$ culture tubes, borosilicate glass (VWR, catalog number: 47729-580)

4. $15 \mathrm{ml}$ conical tubes (Denville Scientific, catalog number: C1018-P)

5. $50 \mathrm{ml}$ conical tubes (Denville Scientific, catalog number: C1062-P)

6. Polycarbonate centrifuge tubes (Beckman Coulter, catalog number: 349622)

7. $4.5 \mathrm{ml}$ polystyrene cuvettes, square (Brookhaven Instruments Corps., catalog number: BI-SCP)

8. $200 \mathrm{~nm}$ polycarbonate membrane (Millipore, catalog number: GTTP01300)

9. Formvar ${ }^{\circledR}$-coated copper grids (Electron Microscopy Sciences, catalog number: FCF300-CU$\mathrm{SC})$

10. Filter paper (VWR, catalog number: 28313-068)

11. Mica sheet (Electron Microscopy Sciences, catalog number: 71855-15)

12. Kimwipes (Kimberly-Clark, catalog number: 06-666)

13. Capillary cell and plastic cap (Malvern, catalog number: DTS 1070)

14. Mouse (Jackson Laboratory, model: FVB/NJ 001800)

15. Phosphate-buffered Saline (PBS) (Corning, catalog number: 21-040-CV)

16. Methanol (Sigma-Aldrich, catalog number: $34860-1 \mathrm{~L}-\mathrm{R}$ )

17. Dichloromethane (Sigma-Aldrich, catalog number: 650463-1L)

18. HEPES buffer pH 7.4 (Sigma-Aldrich, catalog number: F7876)

19. Negative control siRNA (Santa Cruz, catalog number: sc-37007)

20. Positive CD98 siRNA (Santa Cruz, catalog number: sc-35033)

21. Glucose (Sigma-Aldrich, catalog number: 49163)

22. Turbofect ${ }^{\mathrm{TM}}$ reagent (Thermo Fisher Scientific, catalog number: R0531)

23. Potassium chloride ( $\mathrm{KCl}$, Millipore, catalog number: 7447-40-7)

24. Quant-iT Ribogreen reagent (Invitrogen, catalog number: R11491)

25. Uranyl acetate (Electron Microscopy Sciences, catalog number: 22400-4)

26. DiR dye (DilC 18 (7)) (Thermo Fisher Scientific, catalog number: D12731)

27. Amicon ${ }^{\circledR}$ ultra-15 centrifugal filter (MWCO 10,000) (Sigma-Aldrich, catalog number: UFC 9100)

28. $1 \%$ uranyl acetate solution (see Recipes)

29. $5 \%$ glucose solution (see Recipes) 


\section{Equipment}

1. Pipettes: $0.5-10 \mu \mathrm{l}, 10-100 \mu \mathrm{l}$ and 100-1,000 $\mu \mathrm{l}$ (Fisher Scientific, Eppendorf, catalog number: 13-684-251)

2. Particle size analyzer (Brookhaven Instrument Corp., model: 90Plus)

3. Zetasizer (Malvern, model: Nano-ZS90)

4. Atomic force microscopy instrument (Seiko Instrument Inc., model: SPA400)

5. Transmission electron microscope (Carl Zeiss, model: LEO 906E)

6. Milli-Q advantage A10 water purification system (Millipore-sigma, catalog number: C10117)

7. Centrifuge (Thermo Fisher Scientific, model: Sorvalis ST16R)

8. Ultracentrifuge (Beckman Coulter, model: Optima L-90K)

9. $-80{ }^{\circ} \mathrm{C}$ freezer (So-Low, model: PV85-21)

10. Sonicator (Branson, model: 3510 )

11. Vortexer (Scientific Industries, model: 200-SI0236)

12. Rotary evaporator (Buchi, model: R-210)

13. Vacuum pump (Buchi, model: V-700)

14. Vacuum controller (Buchi, model: V-800)

15. Heating bath (Buchi, model: B-491)

16. Evaporating flask (Buchi, catalog number: Z402982)

17. Liposome extruder (Avanti Polar Lipids, model: Mini extruder)

18. Microplate reader (BioTek, Model: Synergy2)

19. IVIS in vivo imaging system (PerkinEImer, model: IVIS Spectrum Series)

\section{Procedure}

A. Extraction of lipids from ginger derived nanoparticles (GDNPs)

1. Prepare $1.6 \mathrm{ml}$ of purified GDNPs PBS suspension (1 mg/ml, refer to Sung et al., 2019).

2. Add $6 \mathrm{ml}$ of methyl alcohol/dichloromethane $(2: 1)(\mathrm{v} / \mathrm{v})$ to $1.6 \mathrm{ml}$ GDNPs suspension in a $22 \mathrm{ml}$ glass culture tube and shake thoroughly.

3. Add $2 \mathrm{ml}$ of dichloromethane and $2 \mathrm{ml}$ of $\mathrm{ddH}_{2} \mathrm{O}$ sequentially then briefly vortex the mixture.

4. Centrifuge the solution in a glass tube at $2,000 \times \mathrm{g}$ for $10 \mathrm{~min}$ at room temperature (RT) for separation of aqueous and organic phases (Figure 1). 


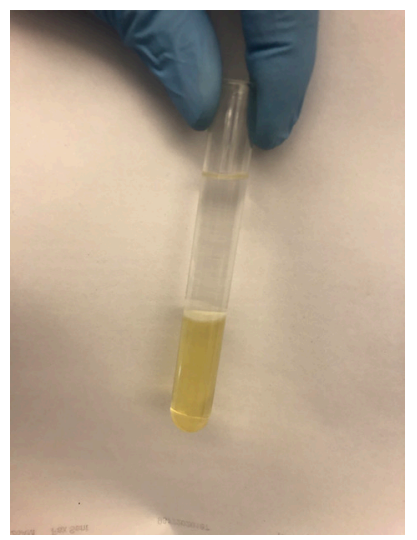

Figure 1. A representative image of liquid-liquid extraction to isolate lipids from GDNPs

5. Remove the upper aqueous phase by pipetting.

6. Collect the remaining GDNPs extracted lipid solution into a new glass culture tube by pipetting.

7. Wash the collected samples with $0.5 \mathrm{ml}$ of $1 \mathrm{M} \mathrm{KCl}$ and centrifuge at 2,000 $\times \mathrm{g}$ for $10 \mathrm{~min}$ then remove the aqueous phase. Wash again with $0.5 \mathrm{ml}$ of $\mathrm{ddH}_{2} \mathrm{O}$ with the same condition.

Note: The wash step generally includes: add the solvent, vortex for $2 \mathrm{~min}$, and centrifuge at $2,000 \times \mathrm{g}$ for $10 \mathrm{~min}$ (RT).

8. Approximately $4-5 \mathrm{ml}$ of total lipids solution is acquired, and it can be stored at $-20^{\circ} \mathrm{C}$ for two weeks or continue to the next step.

9. Transfer the $5 \mathrm{ml}$ lipid solution to a $250 \mathrm{ml}$ eggplant shape flask.

10. Put the flask on a rotary evaporator for $5-10 \mathrm{~min}$ to remove the organic phase $\left(50^{\circ} \mathrm{C}\right)$ and to form an ultrathin ginger lipids film on the wall of the eggplant shape (Figure 2).

Note: Appropriate rotation speed is 4 (out of 10).

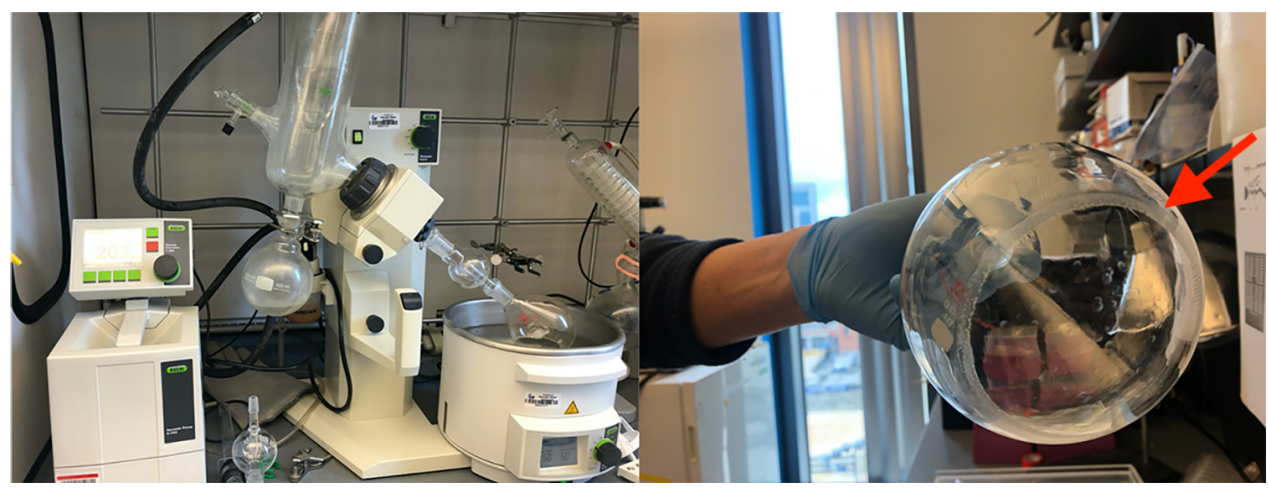

Figure 2. Formation of the thin film of lipids (the red arrow indicates) on the bottom surface of eggplant shaped flask by rotary evaporation

B. Generating ginger derived lipid vesicles (GDLVs) siRNA complex by thin-film hydration

1. Dissolve $3.75 \mathrm{nmol}$ of target siRNA or negative control-siRNA into $300 \mu \mathrm{l}$ of sterile $5 \%$ glucose solution in separate flasks and vortex lightly (10 s). 
2. Add $6 \mu \mathrm{l}$ TurboFect ${ }^{\mathrm{TM}}$ reagent to the siRNA glucose solution, vortex lightly (10 s), and incubate for $15 \mathrm{~min}$ at RT.

3. Add the siRNA/TurboFect glucose solution to the flask which contains the ginger lipids film (1.6 mg GDNPs).

4. Add $500 \mu \mathrm{l}$ of $20 \mathrm{mM}$ HEPES buffer ( $\mathrm{pH} 7.4)$.

5. Sonicate (at $\sim 42 \mathrm{KHz}$ ) the flask with pipetting for $5 \mathrm{~min}$ at RT.

6. Add $500 \mu \mathrm{l}$ of $20 \mathrm{mM}$ HEPES buffer ( $\mathrm{pH} 7.4)$.

7. Sonicate the suspension for another $5 \mathrm{~min}$ (Figure 3).

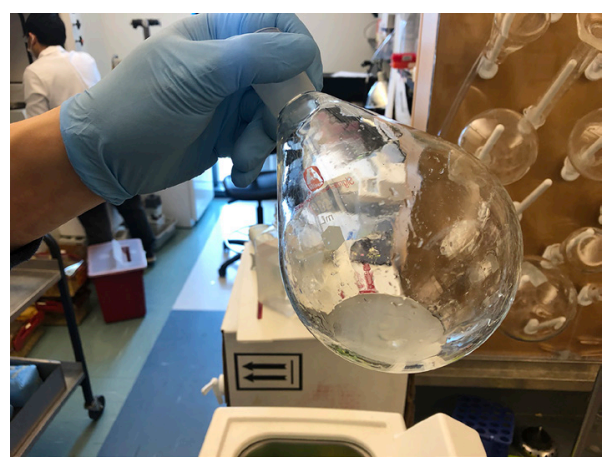

Figure 3. Formation of GDLVs/siRNA complex after sonication

8. Measure the size of the GDLVs/siRNA complex with a size analyzer.

Note: Use 1x PBS (RT) for accurate size measurement.

9. Squeeze the suspension through AVESTIN liposomes extruder (with a $200 \mathrm{~nm}$ polycarbonate membrane) for about $19-21$ times at about $55^{\circ} \mathrm{C}$.

10. Suspend the nanoparticles with $5 \mathrm{ml}$ of autoclaved $1 \mathrm{x}$ PBS for the next step or store it at $-80^{\circ} \mathrm{C}$ for future use (Note 3).

C. Characterization of siRNA-loaded GDNPs (GDLVs/siRNA complex)

\section{siRNA loading efficiency test}

1. Dilute $5 \mu \mathrm{g}$ GDLVs/siRNA complex in TE buffer to a final volume of $1 \mathrm{ml}$.

2. Add $1 \mathrm{ml}$ of Quant-iT RiboGreen reagent.

3. Incubate the solution for $5 \mathrm{~min}$ at RT in the dark condition.

4. Measure the fluorescence of the samples with a microplate reader (excitation $480 \mathrm{~nm}$, emission $520 \mathrm{~nm})$.

\section{Evaluation of the colon-specific targeting (in vivo bio-distribution) of GDLVs}

1. Incubate $5 \mathrm{ml}$ of GDLVs $(0.1-1 \mathrm{mg} / \mathrm{ml}$ in PBS) with DiR dye $(10 \mu \mathrm{M})$ for $30 \mathrm{~min}$ at RT.

2. Transfer the solution to Amicon ${ }^{\circledR}$ ultra-15 centrifugal filter.

3. Centrifuge the filter at $4{ }^{\circ} \mathrm{C}, 5,000 \times g$ for $30 \mathrm{~min}$. 
4. Retrieve the DiR-labeled GDLVs on the apical side of the tube. Reconstitute the DiR-labeled GDLVs in a $5 \mathrm{ml}$ PBS solution.

5. Prepare two groups of $12 \mathrm{~h}$-fasted FVB mice ( 6 weeks old, female, $\mathrm{n}=3$ ).

6. Gavage a single dose of DiR-labeled GDLVs $(0.3 \mathrm{mg} / \mathrm{ml})$ to one group of $12 \mathrm{~h}$-fasted mice at different time points $(4,8,12$, and $24 \mathrm{~h})$.

7. Sacrifice both groups of the mouse and collect tissue samples of various organs (heart, liver, spleen, lung, stomach, small intestine, colon, and kidney).

8. Take ex vivo fluorescence images of the organs using IVIS Spectrum Series in vivo imaging system (fluorescence excitation at $750 \mathrm{~nm}$, emission at $800 \mathrm{~nm}$ ).

9. Compare the fluorescence images between organ samples from mice with and without treatment.

Note: Ideally, the colon tissues will present high fluorescence intensities $\left(>2.5 \times 10^{7}\right.$ radiant efficiency) in 12 and $24 \mathrm{~h}$ samples from mice with DiR-labeled GDLVs.

\section{Testing for delivery of functional siRNA (in vivo transfection)}

1. Gavage $100 \mu \mathrm{l} \mathrm{GDLVs/siRNA} \mathrm{solution}(0.5 \mathrm{nmol} / \mathrm{ml}$ in PBS) twice (12 $\mathrm{h}$ apart) to the mice.

2. After $48 \mathrm{~h}$, sacrifice the mice and collect the intestine tissues for immediate tests or store the tissues at $-80^{\circ} \mathrm{C}$.

3. Perform standard real-time $\mathrm{qPCR}$ for CD98 mRNA with different parts from the intestine, including duodenum, jejunum, ileum, and colon.

4. Compare the CD98 mRNA expressions in different samples from mice treated with or without CD98 siRNA.

Note: A successful knock-down of CD98 mRNA will inhibit more than $80 \%$ of the mRNA expression of CD98 with dosage at 10,000 times lower than naked CD98 siRNA (refer to Zhang et al., 2017).

D. Characterization of GDLVs/siRNA complex

\section{Measure particle size}

1. Dilute $0.2 \mathrm{ml}$ of GDLVs/siRNA complex solution into $1.8 \mathrm{ml}$ PBS.

2. Carefully add the diluted mixture to a cuvette, avoid producing bubbles.

3. Insert the cuvette to the particle analyzer chamber.

4. Determine the size of particles.

\section{Measure zeta potential}

Note: Zeta potential is an important character that reflects the surface chemistry of the particles.

1. Carefully add $0.8 \mathrm{ml}$ of GDLVs/siRNA mixture to a capillary cell.

2. Cover the capillary cell with a plastic cap.

3. Insert the cell to the chamber of zeta potential analyzer.

4. Measure the zeta-potential value at a neutral $\mathrm{pH}$. 


\section{Acquire transmission electron microscopy (TEM) image}

1. Directly deposit $1 \mu \mathrm{l}$ of GDLVs/siRNA sample onto the surface of a formvar-coated grid.

2. Add $5 \mu \mathrm{l} 1 \%$ uranyl acetate on top of the sample and wait for $15 \mathrm{~s}$.

3. Carefully absorb the sample and uranyl acetate with a disposable wiper without touching the surface.

4. Let the sample dry for $30 \mathrm{~min}$ at RT.

5. Scan the sample with a transmission electron microscope.

\section{Acquire atomic force microscopy (AFM) image}

1. Deposit $5 \mu$ l of GDLVs/siRNA sample to the mica sheet.

2. Dry the sample for $2 \mathrm{~h}$ (at RT).

3. Gently rinse the mica sheet three times each with $20 \mu \mathrm{l}$ of distilled water.

4. Dry the sample at RT for another $2 \mathrm{~h}$.

5. Leave it for $\mathbf{3 0} \mathrm{min}$ at RT until the sample becomes flat.

6. Scan the sample with an area of about $4 \times 4 \mu \mathrm{m}$ and $2-50 \mathrm{~nm}$ in height.

Note: To judge whether a sample is dried and becomes flat, we can place the mica sheet to a vertical position, and if we observe no sign of flow from the sample spot, it generally means that the sample is flat and dried.

\section{Data analysis}

1. TEM and AFM images show that the GDLVs are nano-sized particles and have the spherical shape (Images can be found in Figure 2 from Zhang et al., 2017).

2. Dynamic light scattering analysis showed that it has about $189.5 \mathrm{~nm}$ in diameter and zeta potential varies from -18.1 to $-18.4 \mathrm{mV}$ (Related data can be found in supplementary data from Zhang et al., 2017).

3. The efficiency of siRNA loading is calculated according to a standard curve provided by the Quant-iT RiboGreen manufacturer. Fluorescence of samples is measured using standard fluorescence wavelength, with the excitation at $480 \mathrm{~nm}$ and the emission at $520 \mathrm{~nm}$.

\section{Notes}

1. It is recommended to use HPLC purified siRNA because they are more stable and have less toxicity when administered to animal models.

2. Clean the eggplant shaped flask with $100 \%$ ethanol and completely remove the remaining liquid to prevent disruption of nanoparticle formation.

3. Stored nanoparticle (in $1 \times \mathrm{PBS}$ ) can be enriched after ultracentrifugation $\left(30,000 \times \mathrm{g}, 4^{\circ} \mathrm{C}\right.$, and $45 \mathrm{~min}$ ) and removal of the supernatant. 


\section{Recipes}

1. $1 \%$ uranyl acetate solution

a. Dissolve $4 \mathrm{~g}$ uranyl acetate powder into $100 \mathrm{ml}$ preheated $\left(50-60{ }^{\circ} \mathrm{C}\right)$ filtered water to prepare $4 \%$ uranyl acetate stock solution

b. Next, dilute $1 \mathrm{ml} 4 \%$ uranyl acetate stock solution into $3 \mathrm{ml}$ of filtered water

2. $5 \%$ glucose solution

Dissolve $1 \mathrm{~g}$ glucose in $200 \mathrm{ml}$ of $\mathrm{ddH}_{2} \mathrm{O}$ and mix well

\section{Acknowledgments}

This work was supported by the National Institute of Health of Diabetes and Digestive and Kidney (RO1-DK-071594 to D.M.). This work was adapted and modified from Zhang et al., 2017.

\section{Competing interests}

The authors declare no conflicts of interest.

\section{Ethics}

All the experiments conducted involving mice were approved by institutional animal care and use committee (IACUC) at Georgia State University (Atlanta, GA, USA).

\section{$\underline{\text { References }}$}

1. Nikam, R. R. and Gore, K. R. (2018). Journey of siRNA: clinical developments and targeted delivery. Nucleic Acid Ther 28(4): 209-224.

2. Sung, J., Yang, C., Viennois, E., Zhang, M., and Merlin, D. (2019). Isolation, purification, and characterization of Ginger-derived Nanoparticles (GDNPs) from Ginger, Rhizome of Zingiber officinale. Bio-protocol 9(19): e3390.

3. Tatiparti, K., Sau, S., Kashaw, S. K., and lyer, A. K. (2017). siRNA delivery strategies: a comprehensive review of recent developments. Nanomaterials (Basel) 7(4).

4. Zhang, M., Viennois, E., Prasad, M., Zhang, Y., Wang, L., Zhang, Z., Han, M. K., Xiao, B., Xu, C., Srinivasan, S. and Merlin, D. (2016a). Edible ginger-derived nanoparticles: A novel therapeutic approach for the prevention and treatment of inflammatory bowel disease and colitis-associated cancer. Biomaterials 101: 321-340.

5. Zhang, M., Viennois, E., Xu, C., and Merlin, D. (2016b). Plant derived edible nanoparticles as a new therapeutic approach against diseases. Tissue Barriers 4(2): e1134415. 
6. Zhang, M., Wang, X., Han, M. K., Collins, J. F., and Merlin, D. (2017). Oral administration of ginger-derived nanolipids loaded with siRNA as a novel approach for efficient siRNA drug delivery to treat ulcerative colitis. Nanomedicine (Lond) 12(16): 1927-1943. 